\title{
Mechanism of Roux-en-Y Gastric Bypass Treatment for Type 2 Diabetes in Rats
}

\author{
Hongwei Yu • Xiyan Zheng • Zongming Zhang
}

Received: 5 December 2012 / Accepted: 18 March 2013 /Published online: 12 April 2013

(C) 2013 The Author(s). This article is published with open access at Springerlink.com

\begin{abstract}
Objectives Roux-en-Y gastric bypass (RYGB) is a novel therapy for diabetes. We aimed to explore the therapeutic mechanism of RYGB.

Methods After RYGB, animal models were established, and gene expression profile of islets was assessed. Additionally, gastrointestinal hormones were measured using enzyme-linked immunosorbent assays. $\mathrm{Ca}^{2+}$ was studied using confocal microscopy and patch-clamp technique. The morphology of islets and beta cells was observed using optical microscopy and electron microscopy.

Results RYGB was an effective treatment in diabetic rats. Expression profiling data showed that RYGB produced a new metabolic environment and that gene expression changed to adapt to the new environment. The differential expression of genes associated with hormones, $\mathrm{Ca}^{2+}$ and cellular proliferation was closely related to RYGB and diabetes metabolism. Furthermore, the data verified that RYGB led to changes in hormone level and enhanced $\mathrm{Ca}^{2+}$ concentration changes and $\mathrm{Ca}^{2+}$ channel activity. Morphological data showed that RYGB induced the proliferation of islets and improved the function of beta cells.

Conclusions RYGB promoted a new metabolic environment while triggering changes to adapt to the new environment. These changes promoted the cellular proliferation of islets and improved the function of beta cells. The quantity of beta cells increased, and their quality improved, ultimately leading to insulin secretion enhancement.
\end{abstract}

Keywords Roux-en-Y gastric bypass · Islets $\cdot$ Insulin $\cdot$ Beta cells · Gene expression profile

\section{Introduction}

Diabetes has become an epidemic disease in the twenty-first century. The International Diabetes Federation estimated

This study was supported by project grants to ZM Zhang from the National Natural Science Foundation of China (81071996), the Fund of Capital Medical Development and Research (no. 2009-1020) and Tsinghua-YueYuen Medical Science Foundation (nos. 20240000531 and 20240000568).

Electronic supplementary material The online version of this article (doi:10.1007/s11605-013-2188-3) contains supplementary material, which is available to authorized users.

$\mathrm{H}$. Yu $\cdot$ X. Zheng $\cdot$ Z. Zhang $(\bowtie)$

School of Medicine, Tsinghua University, Beijing, People's

Republic of China

e-mail: zhangzongming@mail.tsinghua.edu.cn

Z. Zhang

Department of Hepatobiliary Surgery, Futian Hospital, Guangdong

Medical College, Shenzhen, People's Republic of China that the global diabetes prevalence was 285 million in 2010. ${ }^{1}$ Moreover, over 4 million people die due to complications associated with the disease every year. Although initiatives should be pursued to prevent the onset of type 2 diabetes, effective treatment must be available for people who develop type 2 diabetes. ${ }^{2}$ Traditional treatment modalities for diabetes do not satisfactorily control the disease or its complications, ${ }^{3}$ and current emerging treatments remain in the exploratory or extension phase. Therefore, discovering new treatments has been the focus of considerable medical research. Roux-en-Y gastric bypass (RYGB) is a novel approach among the available bariatric surgery procedures and has been utilized in type 2 diabetes with a good outcome, ${ }^{4,5}$ but the therapeutic mechanisms have remained unclear, and various hypotheses have been debated..$^{6-9}$ Surgical indication has been restricted, and the surgical efficacy is not completely satisfactory. ${ }^{10}$ Therefore, studying the mechanism through which RYGB alleviates type 2 diabetes is a critical theoretical and clinical research endeavor. Our study explored the therapeutic mechanism of RYGB in diabetes using an animal model. In this study, we investigated the impact of RYGB on not only diabetic rats but also 
healthy rats. By analyzing the similarities and differences in the impact of RYGB on diabetic rats and healthy rats, we found that RYGB promoted the cellular proliferation of islets and improved the function of beta cells, ultimately leading to insulin secretion enhancement.

\section{Materials and Methods}

\section{Experimental Animals}

Five-week-old male Wistar and Goto-Kakizaki rats were purchased from SLAC Laboratory Animals Co. Ltd. (Shanghai, China). Wistar rats were chosen as the healthy rat model, and Goto-Kakizaki rats were chosen as the non-obese type 2 diabetic rat model. GotoKakizaki rats are spontaneous diabetic rats that were developed by the selective inbreeding of Wistar rats. ${ }^{11,12}$ All rats were treated according to the approved protocols of the Tsinghua University Animal Care and Use Committee. Wistar rats $(n=20)$ were randomly assigned to a control cohort of healthy rats (WT, $n=10$ ) or an operation cohort of healthy rats (WTO, $n=10$ ). GotoKakizaki rats $(n=20)$ were also randomly assigned to a control cohort of type 2 diabetic rats $(\mathrm{GK}, n=10)$ or an operation cohort of type 2 diabetic rats $(\mathrm{GKO}, n=10)$. After the rats had adapted to the environment for 1 week, the operation cohorts underwent RYGB. ${ }^{13-15}$ The rats were anesthetized with $1 \%$ pentobarbital sodium $(30 \mathrm{mg} / \mathrm{kg})$ through intraperitoneal injection. The RYGB procedure included several key steps. (1) Approximately $20 \%$ of the original gastric volume was preserved. (2) The distal jejunum was connected via an end-to-end anastomosis to the preserved stomach after the jejunum was transected $8 \mathrm{~cm}$ distal to the ligament of Treitz. (3) The proximal jejunum was connected via an end-to-side anastomosis to the jejunum $10 \mathrm{~cm}$ distal to the gastrojejunal anastomosis.

\section{Pancreatic Islet Isolation and Beta Cell Culture}

1. Pancreatic islet isolation: The rats were dissected 4 weeks after RYGB, and the islets were isolated. First, the pancreaticobiliary duct was retrograde intubated and clamped. Next, $10 \mathrm{ml}$ collagenase $\mathrm{V}$ was infused in the duct. Then, the pancreas was resected and digested for $25 \mathrm{~min}$. After the digestion was terminated, single islets were isolated under a stereomicroscope. ${ }^{16}$

2. Beta cell culture: The islets were collected in a centrifuge tube, and a 3-ml trypsin solution was added. Then, the centrifuge tube was gently shaken for $3 \mathrm{~min}$. Next, $1 \mathrm{ml}$ of media was added to the tube. The centrifuge tube was centrifuged at $500 \mathrm{rpm}$ for $5 \mathrm{~min}$. Then, the supernatant was removed. Finally, $2 \mathrm{ml}$ of culture medium was added to the centrifuge tube. The medium containing $60-70 \%$ beta cells was transferred to a dish, and the cells were cultured in an incubator.

Measurement of Blood Glucose, Food Intake, and Body Weight

At 4 weeks after RYGB, the rats were fasted for $6 \mathrm{~h}$, their blood glucose was measured by the electrochemical method, and their body weight was measured using an electronic balance. The 24-h spontaneous food intake of rats was measured on three consecutive days using an electronic balance 4 weeks after RYGB.

\section{Expression Profiling}

All GeneChips were processed at the CapitalBio Corporation (Beijing). For gene expression analyses, a total of 600 (60 islets/rat, $n=10$ ) freshly isolated islets were collected from each cohort. First, total RNA was extracted from each cohort sample using Trizol reagent. Then, the RNA concentration was estimated by measuring the absorbance at $260 \mathrm{~nm}$. The RNA quality was verified by electrophoresis on ethidium bromide-stained $1.5 \%$ agarose gels and A260 nm/A280 nm ratios $>1.8$. A total of $500 \mathrm{ng}$ of total RNA was synthesized as the transcription cRNA probe in vitro using the BioArray ${ }^{\text {TM }}$ High Yield ${ }^{\text {TM }}$ RNA Transcript Labeling Synthesis Kit (Affymetrix) according to the manufacturer's instructions. Next, hybridizations were set up. cDNA targets labeled with Cy5 (experimental) and $\mathrm{Cy} 3$ (reference) were combined and hybridized to microarrays for 14 to $18 \mathrm{~h}$ (overnight) at $42{ }^{\circ} \mathrm{C}$. Following hybridization and washing, the membranes were scanned using a Storm 840 Scanner (Molecular Dynamics). ${ }^{17}$ Differentially expressed genes were selected based on an analysis of the fold change. The selection criteria for differentially expressed genes included a $\log$ ratio $\geq 2.0$ for an upregulated gene and a $\log$ ratio $\leq 0.5$ for a downregulated gene. The molecular function and biological processes of differentially expressed genes were analyzed using Molecule Annotation System 3.0 (MAS 3.0). The Gene Chip Rat Genome 2302.0 Array was purchased from Affymetrix (USA).

\section{Measurement of Hormone Levels}

At 4 weeks after RYGB, hormone levels were measured at room temperature using ELISA kits and a multilabel reader (EnVision, PerkinElmer). After the rats were fasted for $6 \mathrm{~h}$, blood was collected from the orbital venous plexus. The tubes for blood samples of glucagon-like peptide 1 (GLP- 
1) contained $10 \mu$ dipeptidyl dipeptidase IV inhibitor. After blood samples stood for $30 \mathrm{~min}$, the tubes were centrifuged to obtain the serum, and the hormone levels were measured. Rat insulin, ghrelin, and active GLP-1 (7-36) ELISA kits were purchased from Millipore (Billerica MA, USA).

Measurement of Intracellular Calcium and Insulin Concentration

At 4 weeks after RYGB, the fluorescence intensities of beta cells were measured using confocal microscopy (Zeiss 710 META, GER). ${ }^{18}$ The cells were seeded in a dish 1 day before the experiment. After loading the cells with $2 \mu \mathrm{mol} / \mathrm{L}$ fluo-4/AM and $2 \mu \mathrm{mol} / \mathrm{L}$ FITC in RPMI-1640 for $30 \mathrm{~min}$, they were washed three times with Hanks solution. Then, the cells were bathed in RPMI-1640 with $8 \mathrm{mmol} / \mathrm{L}$ glucose solution, and measurements were obtained. Beta cells showed red fluorescence in the FITC channel and green fluorescence in the fluo-4/AM channel. The $\mathrm{Ca}^{2+}$ and insulin fluorescence intensities were recorded at 20-s intervals $400 \mathrm{~s}$ after fluorescence intensity stabilization. $F_{0}$ was the initial value of fluorescence intensity after fluorescence intensity stabilization. Changes in the $\mathrm{Ca}^{2+}$ and insulin fluorescence intensity $(\Delta F)$ were calculated using the following equation: $\Delta F=F_{\mathrm{n}}-F_{\mathrm{n}-1}$. Axio Vision Rel.4.7 software was used to analyze the images of the cells.

\section{Electrophysiology of Calcium Channels}

At 4 weeks after RYGB, whole-cell patch-clamping was performed at room temperature using an EPC-10 patchclamp amplifier and PatchMaster software (HEKA, Germany). Beta cells grown in vitro were perfused with a standard external solution containing the following (in millimoles per liter): $120 \mathrm{NaCl}, 3$ D-glucose, $1 \mathrm{MgCl}_{2}, 4 \mathrm{CsCl}, 20$ TEA.CL, $2.5 \mathrm{CaCl}_{2}$, and 10 Hepes, with the osmolarity adjusted to approximately $310 \mathrm{mOsm}(\mathrm{pH}$ 7.4). This solution was further supplemented with $1 \mu \mathrm{mol} / \mathrm{L}$ tetrodotoxin and $100 \mu \mathrm{mol} / \mathrm{L}$ DIDS. The patch pipettes were pulled from borosilicate glass and fire polished. The pipette resistance ranged between 3 and $5 \mathrm{M} \Omega$ after they were filled with the intracellular solution for voltage-dependent calcium channels (VDCCs) to deplete calcium stores and induce stored operated calcium (SOC) influx. The intracellular solution contained the following (in millimoles per liter): 10 Hepes, $125 \mathrm{CsCl}, 1 \mathrm{MgCl}_{2}$. 10 EGTA (pH 7.2) , and $2 \mu \mathrm{mol} / \mathrm{L} \mathrm{TG}$. In all whole-cell experiments, the recording began with a series of resistance values below $20 \mathrm{M} \Omega$. Cells with a capacitance of $>5 \mathrm{PF}$ and a diameter of 11-12 $\mu \mathrm{m}$ were most likely to be pancreatic beta cells. ${ }^{19}$ Voltage-dependent currents were corrected for linear leakage and residual capacitance using on-line $\mathrm{P} / \mathrm{n}$ subtraction. The beta cells were held at $-70 \mathrm{mV}$. For the normalized current recordings, the peak currents were measured at $0 \mathrm{mV}$. For the detection of SOC influx, voltage ramps of $100 \mathrm{~ms}$ spanning a range of -100 to $100 \mathrm{mV}$ were delivered from a holding potential of $0 \mathrm{mV}$ every $2 \mathrm{~s}$ over $600-1,000 \mathrm{~s}$. A maximal current of $-100 \mathrm{mV}$ was used for the statistical analysis. ${ }^{20}$ All voltages were corrected for a liquid-junction potential of $13 \mathrm{mV}$. Capacitance currents were determined and automatically compensated for using the EPC-10 amplifier. Data were analyzed using IGOR Pro 6.20 (Wavemetrics, USA).

\section{Morphology Techniques}

1. At 4 weeks after RYGB, small samples of fresh pancreatic tissue were collected from the pancreas of each rat. Then, the pancreatic tissue was fixed, embedded in paraffin, and continuously cut into 5 - $\mu \mathrm{m}$ sections. After sections that were a certain distance apart were selected, the sections were stained with $\mathrm{HE}$ and imaged with light microscopy (IX71-A12FL/PH Olympus, Japan).

2. At 4 weeks after RYGB, a total of 300 (30 islets/rat, $n=$ 10) freshly isolated islets were isolated from each cohort and conventionally fixed. Then, the islets were dehydrated in ethanol, embedded in Epon-Araldite, strained with toluidine blue, and cut into ultrathin sections. The samples were observed with transmission electron microscopy (Hitachi 7650, Japan). ${ }^{21}$ ImagePro PluS6.0 software was used to analyze the images.

\section{Reagents}

Collagenase V, FITC, trypsin, and Trizol were purchased from Sigma (USA). Fluo-4/AM was purchased from Invitrogen (USA). Thapsigargin was purchased from Merck (GER).

\section{Statistical Analysis}

Measurement data were expressed as the mean $\pm \mathrm{SD}$, and count data were expressed as percentage differences. The data were analyzed with a repeated-measures oneway ANOVA. $P<0.05$ was considered statistically significant. All statistical analyses were performed using SPSS 18.0 statistical software.

\section{Results}

\section{Effect of RYGB on Correlated Metabolic Parameters}

To evaluate whether the RYGB animal models met the experimental requirements, several basic metabolic parameters (blood glucose, serum insulin, body weight, and food intake) were measured 4 weeks after RYGB. First, comparing the GK cohort with the WT cohort, the blood glucose levels and food intake were higher in the GK cohort; however, the body 
Table 1 Effect of RYGB on correlated metabolic parameters

\begin{tabular}{lrrrr}
\hline & \multicolumn{1}{l}{ WT } & \multicolumn{1}{c}{ WTO } & GK & GKO \\
\hline Glucose (mmol/L) & $6.39 \pm 0.81$ & $6.05 \pm 0.85$ & $23.36 \pm 3.42 * *$ & $10.53 \pm 1.41^{*}$ \\
Food intake (g/d) & $25.32 \pm 1.34$ & $24.01 \pm 1.33$ & $27.23 \pm 1.23 * *$ & $24.81 \pm 1.03 *$ \\
Body weight (g) & $370.00 \pm 4.29$ & $364.20 \pm 3.01$ & $355.70 \pm 9.04 * *$ & $360.70 \pm 2.87$ \\
Insulin (ng/ml) & $2.12 \pm 0.33$ & $5.23 \pm 0.83 * * *$ & $1.20 \pm 0.25 * *$ & $1.89 \pm 0.19 * * *$ \\
\hline
\end{tabular}

Values represent the mean $\pm \mathrm{SD}$ of $n=10$

${ }^{*} P<0.01$, significant difference between GKO and GK; ${ }^{* *} P<0.01$, significant difference between WT and GK; ${ }^{* * *} P<0.01$, significant difference between WTO and WT; ****P<0.05, significant difference between GKO and GK

a

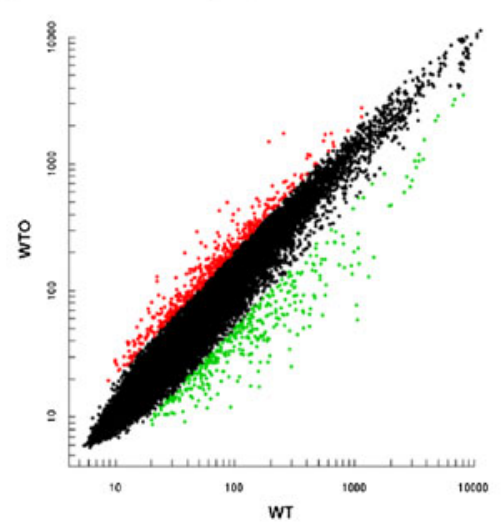

b

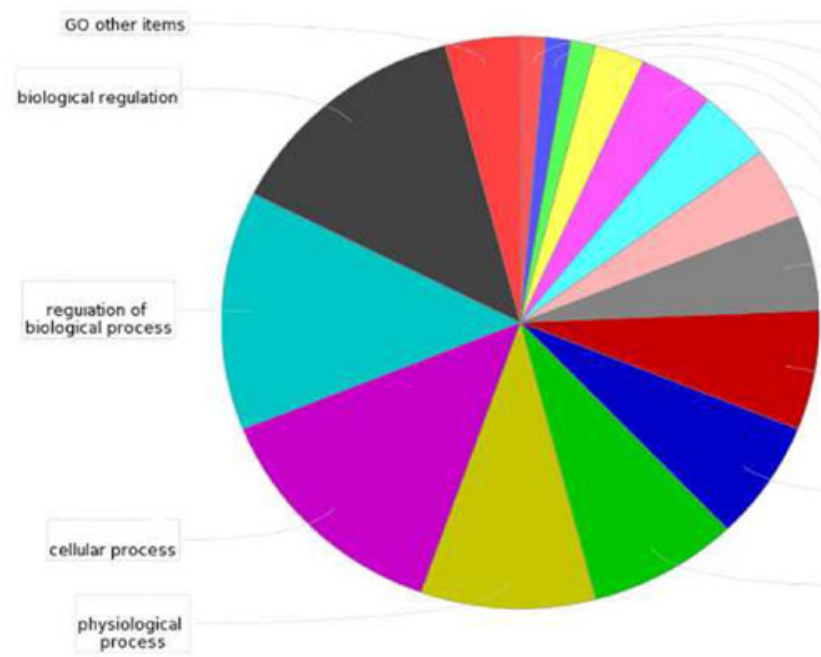

Fig. 1 Effect of RYGB on the gene expression of islets. a Differentially expressed genes in islets were screened by fold analysis. Red plots indicate relatively increased expression ( $\log$ ratio $\geq 2.0$ ), black indicates median expression, and green indicates decreased expression $(\log$ ratio $\leq 0.5)$. b Pie chart of GO mapping illustrates the biological process, molecular function, and cellular component of the differentially expressed genes. Comparing the operation cohorts with the control cohorts, 685 differentially expressed genes were identified. The differentially expressed genes were analyzed using the Molecule Annotation System, and biological regulation, regulation of biological process-, and metabolism-related genes accounted for a large proportion (up to $49.6 \%$ ). Thus, these differentially expressed genes were mainly associated with metabolic regulation. GO mapping

\section{Log-Log Scatter Plot}

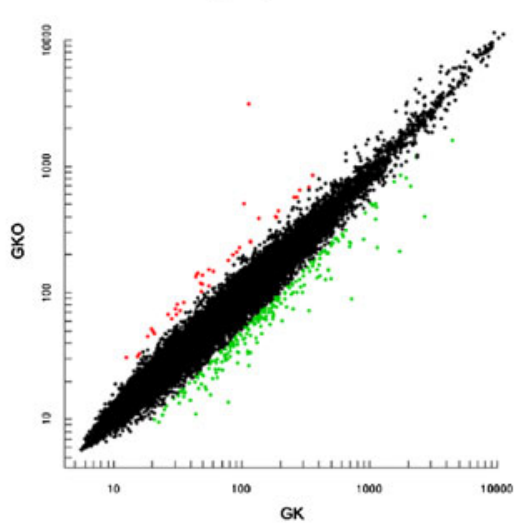

Log-Log Scatter Plot

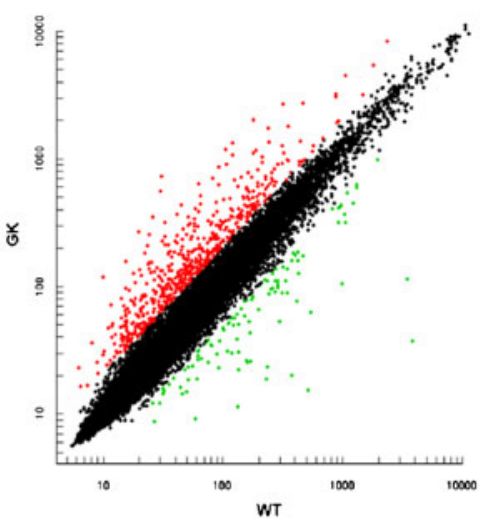

C
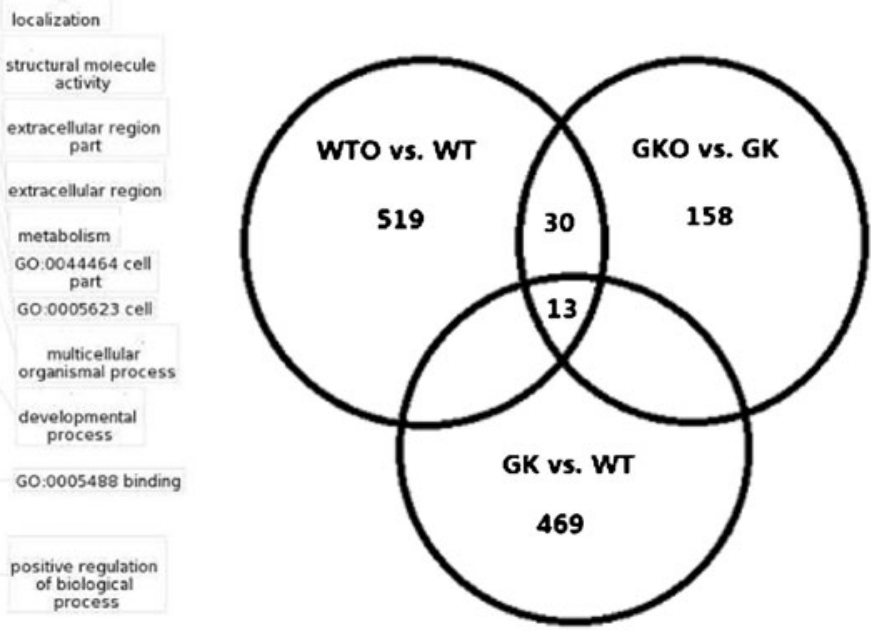

suggested that the RYGB procedure led to the creation of a new metabolic environment, and the expression of genes that were primarily related to metabolic regulation was adjusted to adapt to the new metabolic environment. $\mathbf{c}$ Venn diagram of the differentially expressed genes according to the RYGB procedure and diabetes metabolism. The circle with "519" indicates 519 differentially expressed genes between WTO and WT, and the circle with " 158 " indicates 158 differentially expressed genes between GKO and GK. The intersection of the two circles with " 30 " indicates that the 30 genes were the result of RYGB. The circle with " 469 " indicates 469 differentially expressed genes between GK and WT. The intersection of the three circles with " 13 " indicates that 13 genes were closely related to the RYGB procedure and diabetes metabolism 
Table 2 Effect of Roux-en-Y gastric bypass on hormone levels

\begin{tabular}{lrrrr}
\hline & WT & WTO & GK & GKO \\
\hline Ghrelin (ng/ml) & $2.62 \pm 0.08$ & $1.88 \pm 0.08^{* * *}$ & $4.71 \pm 0.17^{* *}$ & $2.83 \pm 0.16^{*}$ \\
GLP-1 (pM) & $11.62 \pm 1.54$ & $15.67 \pm 1.14^{* * *}$ & $7.15 \pm 0.95^{* *}$ & $18.56 \pm 1.75^{*}$ \\
\hline
\end{tabular}

Values represent the mean $\pm \mathrm{SD}$ of $n=10$

${ }^{*} P<0.01$, significant difference between GKO and GK; $* * P<0.01$, significant difference between WT and GK; $* * * P<0.01$, significant difference between WTO and WT

weight and serum insulin were lower in the GK cohort (Table 1). The data showed that the diabetic rats showed symptoms of diabetes. Then, comparing the GKO cohort with the GK cohort, the blood glucose levels and food intake were lower, and the serum insulin was higher in the GKO cohort, but body weight did not change significantly. The metabolic parameters in the WTO cohort were similar to the WT cohort, except for an increase in serum insulin in the WTO cohort (Table 1). RYGB was observed to alleviate type 2 diabetes in diabetic rats and had little effect on the correlated metabolic parameters in healthy rats 4 weeks after

a

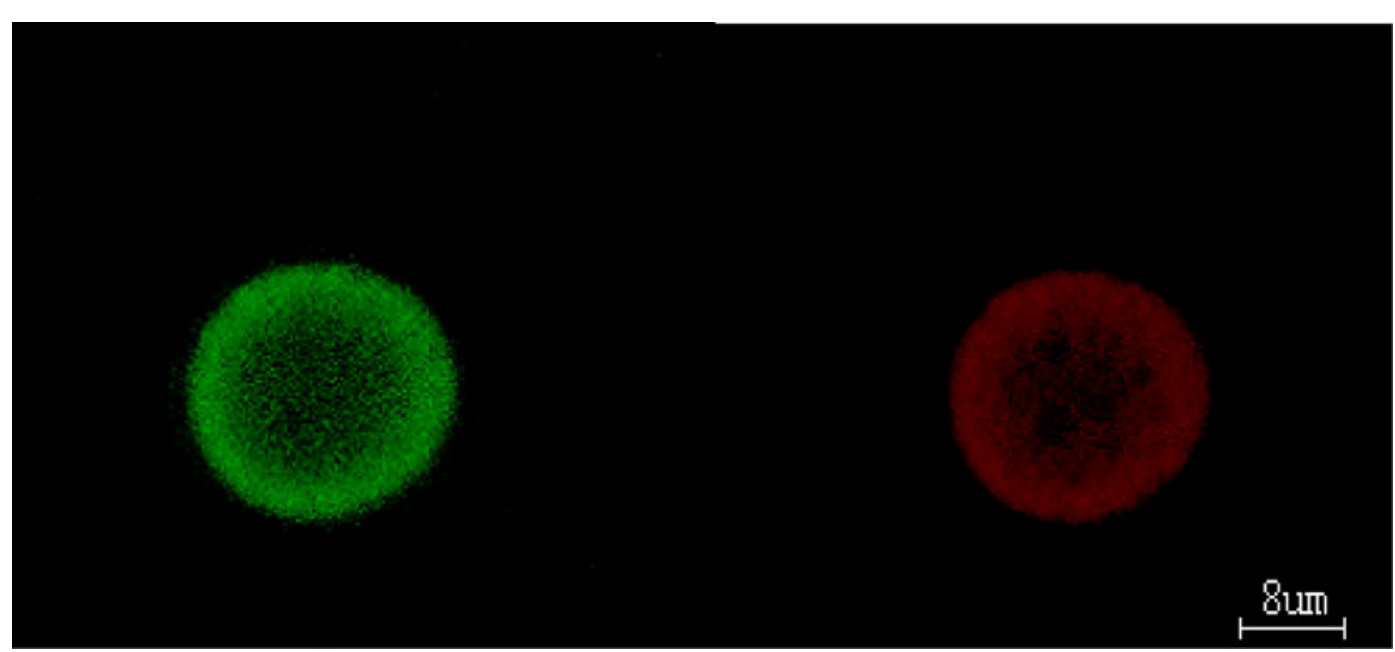

b

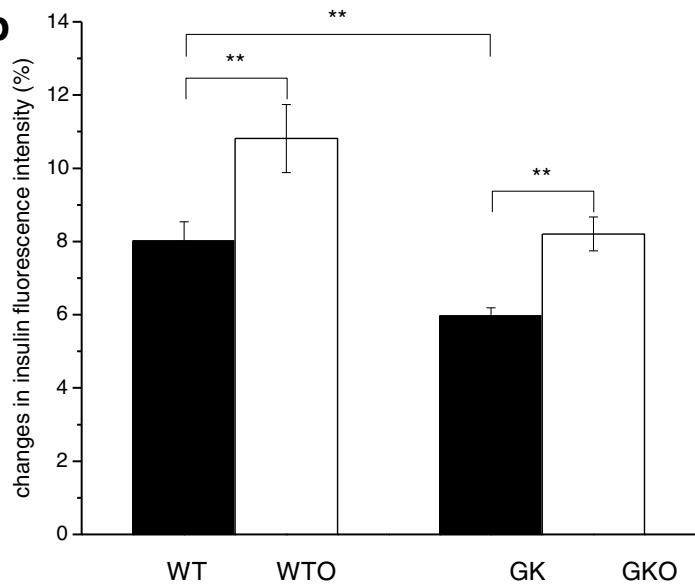

Fig. 2 Effect of RYGB on fluorescence intensity changes of insulin and calcium. a Beta cells were imaged using confocal microscopy after intracellular insulin was labeled with FITC and free $\mathrm{Ca}^{2+}$ was labeled with fluo-4/AM. The red image shows the FITC channel, and the green image shows the fluo-4/AM channel. The scale bars in the images represent $8 \mu \mathrm{m}$. The fluorescence detection channel was set to the

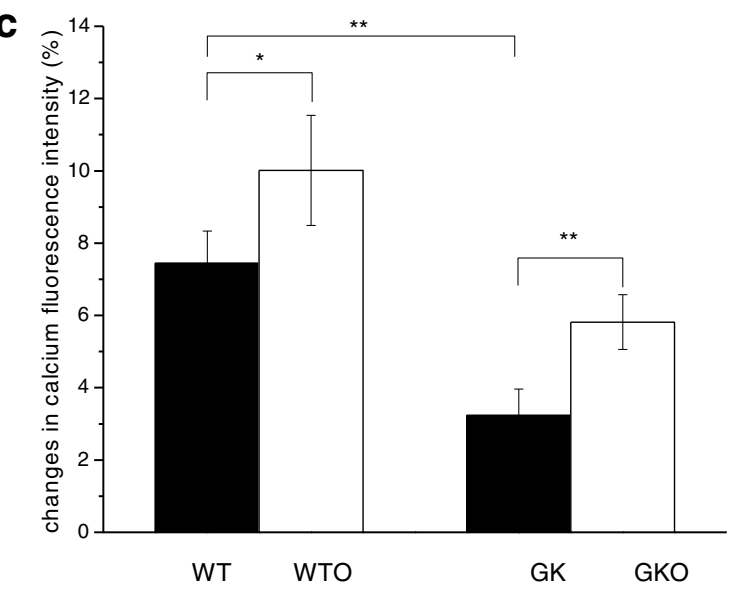

following ranges: fluo-4/AM, 494-516 nm; FITC, 490-495 nm. b Effect of RYGB on fluorescence intensity changes of insulin. $\mathbf{c}$ Effect of RYGB on fluorescence intensity changes of calcium. RYGB increased the fluorescence intensity changes in intracellular insulin and intracellular free $\mathrm{Ca}^{2} . * P<0.05, * * P<0.01$, significant difference 
RYGB. Thus, RYGB animal models could be applied for exploring the therapeutic mechanism of RYGB.

Effect of RYGB on the Gene Expression Profile of Islets

The mechanism of RYGB treatment for diabetes is complex. To identify meaningful clues among the complicated clues, the gene expression profile of islets was analyzed. First, comparing the GKO cohort with the GK cohort and the WTO cohort with the WT cohort, 158 and 529 differentially expressed genes, respectively, were identified (Fig. 1a) based on the RYGB procedure. The differentially expressed genes, including 337 upregulated genes and 348 downregulated genes, were analyzed using MAS 3.0. GO mapping showed that these differentially expressed genes were mainly associated with metabolic regulation because biological regulation-, regulation of biological process-, and metabolism-related genes accounted for a large proportion (up to $49.6 \%$ ) (Fig. 1b). The data suggested that the RYGB procedure led to the creation of a new metabolic environment, and in islets, the expression of genes that were primarily related to metabolic regulation was adjusted to adapt to the new metabolic environment. Furthermore, with regard to the impact of RYGB on Goto-Kakizaki rats and Wistar rats, 30 differentially expressed genes were identified. The 30 genes were the result of the RYGB procedure. Next, to identify which of the 30 genes were associated with diabetes, genes that were differentially expressed between diabetic rats and healthy rats were considered. Comparing the GK cohort with the WT cohort, 469 differentially expressed genes were identified (Fig. 1a) due to differences between diabetic rats and healthy rats. After comprehensive comparison, 13 genes (Amyla, Rab30, Tff2, Ankrd57, B4galt5, Birc3, Ccl2, Cks2, Cxcl10, Hmox1, Mdm2, Pcf1, and Tifa) of the 30 differentially expressed genes were selected (Fig. 1c). The data suggested that the 13 differentially expressed genes were closely related to the RYGB procedure and diabetes metabolism. The biological process and molecular function of the 13 genes were further analyzed by gene ontology. Regarding biological process, the genes primarily participated in processes of metabolism, $\mathrm{Ca}^{2+}$ homeostasis, and the regulation of cellular proliferation.
Regarding molecular functions, the genes were mainly related to growth factor activity, hormone activity, and metal ion binding. The gene expression profile of islets showed that differentially expressed genes related to $\mathrm{Ca}^{2+}$ signaling, cellular proliferation, and hormones were closely associated with the effect of the RYGB procedure and diabetes metabolism.

\section{Effect of RYGB on Gastrointestinal Hormone Levels}

RYGB was observed to change the levels of hormones. Comparing the WT cohort with the GK cohort, the level of ghrelin was higher in the GK cohort. Comparing operation cohorts with control cohorts, the level of ghrelin was higher in control cohorts (Table 2). Thus, RYGB decreased the levels of ghrelin. Comparing the WT cohort with the GK cohort, the level of active GLP-1 (7-36) was higher in the WT cohort. Comparing operation cohorts with control cohorts, the level of GLP-1 was higher in operation cohorts (Table 2). Thus, RYGB increased the level of active GLP-1. Taken together, we found that RYGB changed the levels of hormones 4 weeks after RYGB. RYGB could increase the level of active GLP-1 (7-36) and decrease the level of ghrelin in rats 4 weeks after RYGB.

\section{Effect of RYGB on Intracellular Insulin and Calcium}

RYGB may enhance intracellular insulin secretion and changes in free $\mathrm{Ca}^{2+}$ concentration. When intracellular insulin is labeled with FITC and intracellular free $\mathrm{Ca}^{2+}$ is labeled with fluo-4/AM, the fluorescence intensity of insulin and $\mathrm{Ca}^{2+}$ may indirectly reflect the concentration of intracellular insulin and intracellular free $\mathrm{Ca}^{2+}$ (Fig. 2a). Comparing the GK cohort with the WT cohort, the fluorescence intensity changes in insulin and $\mathrm{Ca}^{2+}$ were higher in the WT cohort [insulin (percentage): WT, $8.02 \pm 0.53$ vs. GK, $5.97 \pm$ $0.22, n=19, P<0.01$; calcium (percentage): WT, $7.44 \pm 0.89$ vs. GK, $3.23 \pm 0.73, n=19, P<0.01$; Fig. $2 \mathrm{~b}, \mathrm{c}]$. The data showed that changes in the concentration of intracellular insulin and $\mathrm{Ca}^{2+}$ in diabetic rats were lower than those in healthy rats. Comparing operation groups with control groups, the fluorescence intensity changes in insulin and

Table 3 Effect of RYGB on the electrophysiology of calcium channels

\begin{tabular}{llccr}
\hline & WT & WTO & GK & GKO \\
\hline L-type $(\mathrm{pA} / \mathrm{pF})$ & $6.01 \pm 2.08$ & $6.62 \pm 2.09$ & $11.23 \pm 3.80^{* *}$ & $11.54 \pm 3.42$ \\
T-type $(\mathrm{pA} / \mathrm{pF})$ & $2.13 \pm 0.57$ & $2.01 \pm 0.50$ & $3.81 \pm 0.90^{* *}$ & $4.10 \pm 0.99$ \\
SOC $(\mathrm{pA} / \mathrm{pF})$ & $7.44 \pm 1.98$ & $10.12 \pm 1.76^{* * *}$ & $9.27 \pm 1.92$ & $14.35 \pm 2.04^{*}$ \\
\hline
\end{tabular}

Values represent the mean $\pm \mathrm{SD}$ of $n=18$

$* P<0.01$, significant difference between GKO and GK; $* * P<0.01$, significant difference between WT and GK; $* * * P<0.01$, significant difference between WTO and WT 
$\mathrm{Ca}^{2+}$ were higher in operation cohorts [insulin (percentage): WTO, $10.81 \pm 0.93$ vs. WT, $8.02 \pm 0.53, n=19, P<0.01$; GKO, $8.20 \pm 0.47$ vs. GK, $5.97 \pm 0.22, n=19, P<0.01$; calcium (percentage): WTO, $10.01 \pm 1.52$ vs. WT, $7.44 \pm 0.89, n=$ $19, P<0.05$; GKO, $5.81 \pm 0.76$ vs. GK, $3.23 \pm 0.73, n=19, P$ $<0.01$; Fig. $2 b, c]$. These results implied that RYGB increased intracellular $\mathrm{Ca}^{2+}$ signaling and improved the function of beta cells 4 weeks after RYGB. A significant correlation at the 0.05 level was found between insulin secretion and intracellular free $\mathrm{Ca}^{2+}$ change after RYGB (WTO, Pearson's correlation coefficient, $r=0.998, P=0.031$; GKO, Pearson's correlation coefficient, $r=0.982, P=0.018$ ).
Fig. 3 Effect of RYGB on calcium channels. a Ramp depolarization potentialactivated voltage-dependent $\mathrm{Ca}^{2+}$ influx. The T-type voltage-dependent $\mathrm{Ca}^{2+}$ channel (VDCC) opened at $-50 \mathrm{mV}$ membrane potential. The L-type VDCC opened with an increase in membrane potential and peaked at $0 \mathrm{mV}$, as observed by the twin peak pattern. b Effect of RYGB on L-type VDCC current density. c Effect of RYGB on T-type VDCC current density. L-type and T-type VDCC current densities in the GK cohort were higher than were those in the WT cohort. RYGB did not alter L-type or T-type VDCC current densities. d The stored operated calcium (SOC) current changed over time to $-100 \mathrm{mV}$. The curve reflects SOC channel activation, deactivation, and inactivation. e Effect of RYGB on SOC current density. RYGB enhanced SOC current density. $* * P<0.01$, significant difference a

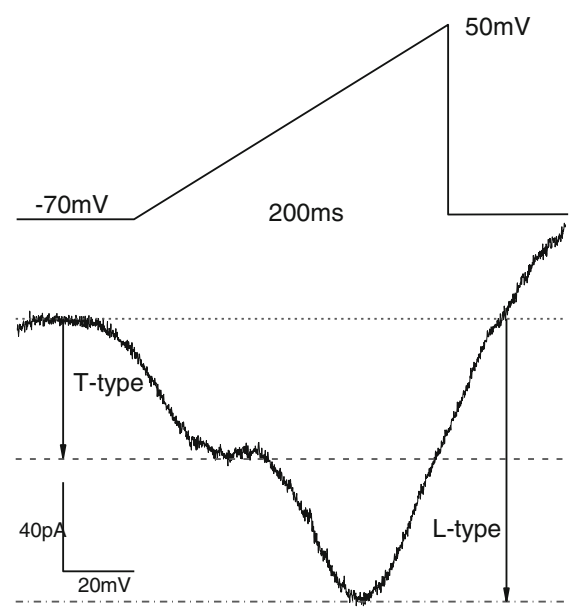

C

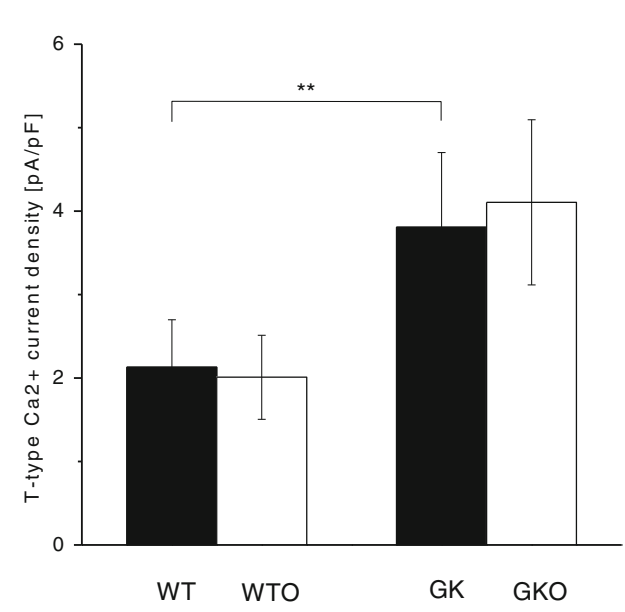

e

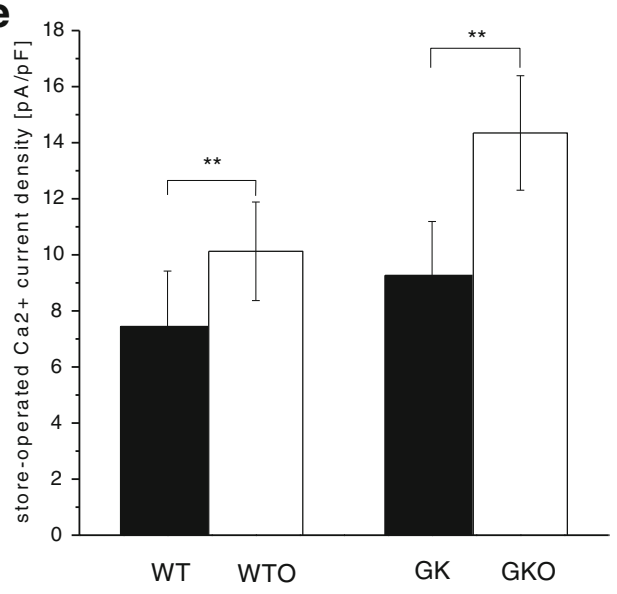

b

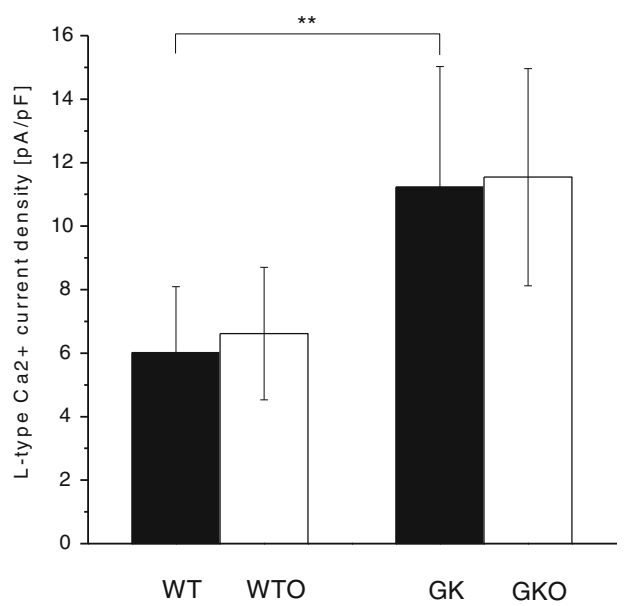

d

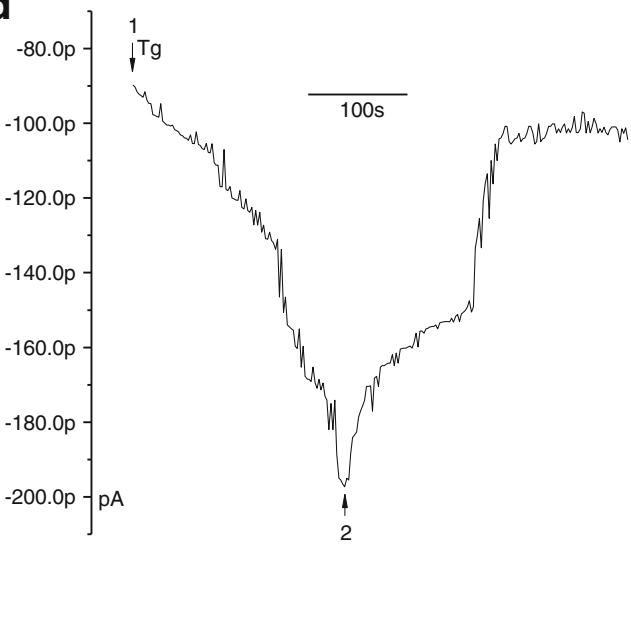


The data showed that intracellular free $\mathrm{Ca}^{2+}$ was closely related to insulin secretion after RYGB. The increase in intracellular free $\mathrm{Ca}^{2+}$ triggered by RYGB played an important role in the functional improvement of beta cells.

\section{Effect of RYGB on Calcium Channels}

RYGB may enhance SOC activity. Comparing the GK cohort with the WT cohort, L-type and T-type VDCC current densities were higher in the GK cohort. L-type and T-type VDCC current densities in the operation cohorts were not significantly different compared to control groups (Table 3, Fig. $3 b, c)$. However, SOC current densities were similar between the WT cohort and the GK cohort. The SOC current densities in the operation cohorts were significantly higher than were those of control cohorts (Table 3, Fig. 3e). RYGB enhanced SOC activity but did not alter VDCC activity 4 weeks after RYGB. Thus, RYGB primarily increased changes in intracellular free $\mathrm{Ca}^{2+}$ through increasing SOC activity.

Effects of RYGB on the Morphology of Islets and Beta Cells

RYGB may promote the differentiation of primitive cells into islets and improve the function of beta-cells. (1) The morphology of islets was studied using optical microscopy. In the WT cohort, the shape of the islets was regular and uniform. The internal structure of the islets was normal, with alpha cells distributed marginally and beta cells distributed centrally. In the GK cohort, the shapes of the islets were irregular and non-uniform. The internal structure of the islets was disorganized. Small islets were occasionally observed. Comparing operation cohorts with control cohorts, a large number of small islets were present in operation cohorts. The small islets only contained $8-24$ cells (two-dimensional cross section), and it was difficult to distinguish the cell type (Table 4, Fig. 4). Thus, RYGB promoted the differentiation of primitive cells into new islets 4 weeks after RYGB. (2) The morphology of beta cells was studied using transmission electron microscopy. Beta cells can store secreted products in vesicles. There were two types of secretory vesicles in beta cells: large dense-core vesicles (LDCVs) and synaptic-like microvesicles. LDCVs are critical for the transport and storage of insulin. The functional ability of beta cells to synthesize and secrete insulin can indirectly be evaluated through the measurement of the area of the vesicles. In the WT cohort, the morphologic features of LDCVs were consistent, and LDCVs were abundant. In the GK cohort, there were few LDCVs in most beta cells. The images showed that most beta cells were dysfunctional. There was no significant difference between the WTO cohort and WT cohort. Comparing the GKO cohort with the GK cohort, it was clear that the secretory vesicles were increased in the GKO cohort. LDCVs were increased by $53.0 \%$ after RYGB (Table 4, Fig. 4). Thus, RYGB improved the function of beta cells 4 weeks after RYGB.

\section{Discussion}

RYGB has a novel therapeutic value for type 2 diabetes. However, the therapeutic mechanism remains unclear. Using an animal model, this study explored the therapeutic mechanism of RYGB in diabetes.

First, an RYGB animal model was established. Then, the therapeutic efficacy of animal model was evaluated. The results confirmed that the model could be applied for exploring the therapeutic mechanism of RYGB.

Next, the therapeutic mechanism of RYGB in diabetes was explored. First, the gene expression profile of islets was analyzed. The gene expression profile can show changes in genome-wide expression. And the physiological dysfunction of islets is the basis of diabetes. Thus, valuable information can be acquired through studying the gene expression profiles of islets. The result showed that after RYGB, a new metabolic environment was present, and the gene expression in islets was changed to adapt to the new metabolic environment. Furthermore, 13 differentially expressed genes that were found were closely related to the RYGB procedure and diabetes metabolism. And the molecular functions and biological process of the genes

Table 4 Effect of RYGB on islets and LDCVs

\begin{tabular}{llllll}
\hline & Small islets & All islets & Small islet ratio (\%) & LDCVs & Percentage \\
\hline WT & 7 & 162 & 4.3 & 268 & - \\
WTO & 32 & 196 & 16.3 & 272 & $1.5 \%$ \\
GK & 19 & 185 & 10.3 & 166 & - \\
GKO & 52 & 212 & 24.5 & 254 & $53.0 \%$ \\
\hline
\end{tabular}

The number of islets is the sum of ten sections for each group. Small islets were selected based on their area. The selection criterion for small islets was area $\geq 1,000 \mu \mathrm{m}^{2}$. The number of LDCVs was calculated in fixed areas and is the sum of ten images for each group

$L D C V_{S}$ large dense-core vesicles, percentage $=($ LDCVs of operation cohort-LDCVs of control cohort $) /$ LDCVs of operation cohort 
Fig. 4 Effect of RYGB on the morphology of islets and beta cells. Sections were observed by OM. The islets were regular in shape and size in the WT cohort. The islets were irregular in shape and size in the GK cohort. At 4 weeks after RYGB, small islets were present in operation cohorts. Ultra-thin sections of islets were viewed by TEM. Secretory vesicles: LDCVs were abundant in beta cells of the WT cohort. LDCVs were relatively scarce in most beta cells of the GK cohort. At 4 weeks after RYGB, LDCVs were more abundant in beta cells of operation cohorts. The scale bars in the images represent $20 \mu \mathrm{m}$ and $500 \mathrm{~nm}$. $O M$ optical microscopy, TEM electron microscopy

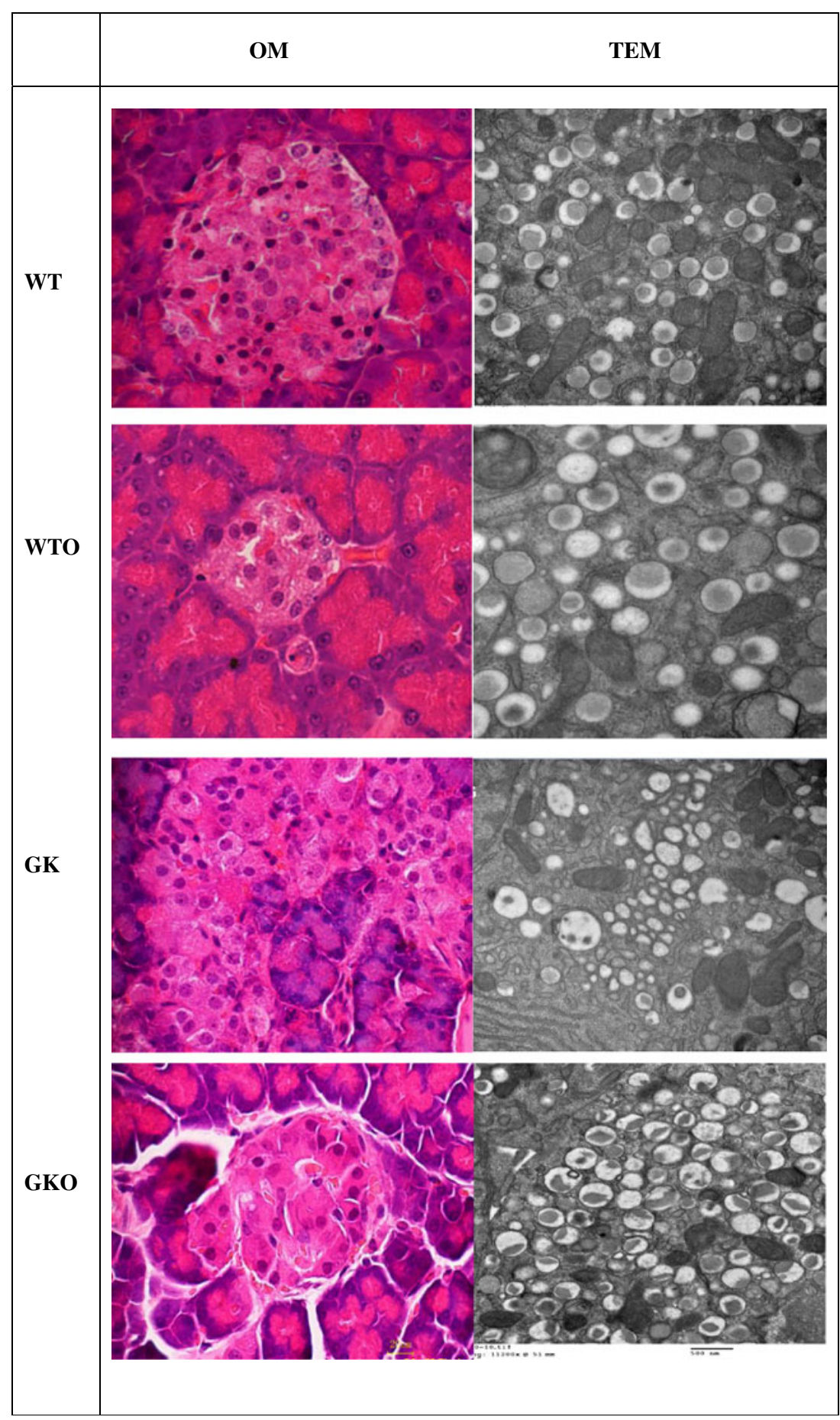

were mainly related to hormones, calcium signals, and cellular proliferation by gene ontology analysis. ${ }^{22-27}$ Further research effects of RYGB on hormones, calcium, and cellular morphology could provide more information.

As messengers that transmit information, hormones play an important regulatory role in the physiological processes of the body. Insulin is an important factor that affects the balance of the body's glucose metabolism. The results showed that RYGB increased serum insulin levels.
Furthermore, HOMA- $\beta$ was used to evaluate the level of insulin secretion. ${ }^{28}$ HOMA- $\beta$ in diabetic rats increased after RYGB. These findings suggested that the underlying therapeutic mechanism of RYGB was enhanced insulin secretion. Previous studies have also reported that RYGB leads to a marked improvement in glucose control, in which gastrointestinal hormones play a role. ${ }^{15}$ Additionally, researchers have proposed various hypotheses regarding gastrointestinal hormones. ${ }^{29,30}$ This study investigated gastrointestinal 
hormones, including ghrelin and GLP-1. Ghrelin is an endogenous ligand of the growth hormone secretagogue receptor. ${ }^{31,32}$ Ghrelin can be expressed in alpha cells and beta cells of islets, and it is not only directly involved in insulin secretion but also determines the differentiation of islet cells. GLP-1 is an important and extensively studied incretin. ${ }^{33,34}$ GLP-1 can stimulate insulin secretion and enhance beta cell proliferation. A synthetic analogue of GLP-1 (exendin-4) has been successfully introduced to treat diabetes patients. ${ }^{35}$ Our results showed that RYGB promoted the secretion of GLP-1 and decreased ghrelin levels. Based on the effects of RYGB on the cellular morphology and hormone levels, and the production location of ghrelin and GLP-1, we speculated that promoting cellular proliferation and improving cellular function are two of the roles that gastrointestinal hormones play in the therapeutic mechanism of RYGB in diabetes.

And previous researchers have proposed that ghrelin and GLP-1 regulate insulin secretion by affecting the calcium signaling pathway. Intracellular free $\mathrm{Ca}^{2+}$ not only triggers secretion ${ }^{36}$ but is also involved in the regulation of exocytosis. ${ }^{37,38}$ Fluorescence labeling may monitor the continuous changes in concentration. Fluo-4 is a highly specific $\mathrm{Ca}^{2+}$ fluorescence probe, and changes in its fluorescence intensity can sensitively reflect changes in the concentration of intracellular free $\mathrm{Ca}^{2+}$. Under appropriate conditions, FITC can bind to insulin. The changes in intracellular fluorescence intensity indirectly indicate the cells' ability to secrete insulin. Labeling calcium and insulin can effectively reflect the calcium signals and insulin secretion in beta cells. The results showed that RYGB increased the concentration of intracellular free $\mathrm{Ca}^{2+}$ and insulin secretion, and the change in intracellular free $\mathrm{Ca}^{2+}$ was closely related to insulin secretion after RYGB. Thus, $\mathrm{Ca}^{2+}$ signal played an important role in the RYGB effect on diabetes. RYGB caused an increase in the concentration of intracellular free $\mathrm{Ca}^{2+}$, which promoted insulin secretion. Our group further studied $\mathrm{Ca}^{2+}$ channels and attempted to elucidate the mechanism through which RYGB increased intracellular free $\mathrm{Ca}^{2+}$. The intracellular free $\mathrm{Ca}^{2+}$ concentration is maintained at a low level. Calcium influx across the cell membrane via a $\mathrm{Ca}^{2+}$ channel increases the $\mathrm{Ca}^{2+}$ concentration within the cytosol. The $\mathrm{Ca}^{2+}$ channels in beta cell membranes are mainly VDCCs, and SOC also exists. ${ }^{39}$ SOC may be involved in $\mathrm{Ca}^{2+}$ signal transmission. ${ }^{40}$ The result showed that RYGB had little effect on VDCC activity. However, RYGB significantly enhanced SOC activity. The finding not only elucidates the mechanism through which RYGB elevated intracellular free $\mathrm{Ca}^{2+}$ but also provides new ideas for the drug treatment of diabetes.

Cellular structure and function are intricately related. After RYGB, the number of small islets and secretory vesicles was increased. The results supported that RYGB could promote the differentiation of primitive cells into islets cells, and improve the function of beta cells. RYGB increased the quantity and improved the quality of insulin secretion cells. These morphological changes were the result of hormone adjustment and calcium signal changes. At the same time, these morphological changes were the basis of insulin secretion improvement.

In summary, we hypothesize that RYGB led to the creation of a new metabolic environment and triggered a series of changes, primarily in hormone levels and $\mathrm{Ca}^{2+}$ signaling, to adapt to this new environment. These adjustments further induced the cellular differentiation of primitive cells into islets cells and improved the function of beta cells. RYGB increased the quantity and improved the quality of insulin secretion cells. RYGB ultimately led to enhancing insulin secretion to treat diabetes. The study of the mechanism through which RYGB alleviates type 2 diabetes is a critical theoretical and clinical research endeavor. The study is a novel approach to determining the mechanism of RYGB. There are more parallel and intersecting comparisons between diabetic rats and healthy rats and the operation cohort and control cohort.

However, the animal model cannot be fully compliant with human type 2 diabetes characteristics. And it is difficult to obtain a large number of high-quality islets. The longterm effects of RYGB have not been explored. Thus, our next study will aim to elucidate the mechanism of long-term effects of RYGB on rats.

Acknowledgments Zongming Zhang reviewed/edited the manuscript. Xiyan Zheng performed experiments and analyzed data. Hongwei Yu analyzed data, wrote the manuscript, and contributed to the discussion.

Open Access This article is distributed under the terms of the Creative Commons Attribution License which permits any use, distribution, and reproduction in any medium, provided the original author(s) and the source are credited.

\section{References}

1. Shaw JE, Sicree RA, Zimmet PZ (2010) Global estimates of the prevalence of diabetes for 2010 and 2030. Diabetes Res Clin Pract 87: 4-14

2. Dixon JB, Zimmet P, Alberti KG, Mbanya JC, Rubino F. Bariatric surgery for diabetes: the international diabetes federation takes a position. J Diabetes 2011;3:261-264

3. Clark NG, Costa F, Eckel R, Fonseca V, Gerstein HC, Grundy S, Nesto RW, Pignone MP, Plutzky J, Porte D, Redberg R, Stitzel KF, Stone NJ. Primary prevention of cardiovascular diseases in people with diabetes mellitus: a scientific statement from the American Heart Association and the American Diabetes Association. Diabetes Care 2007;30:162-172

4. Obeid F, Falvo A, Dabideen H, Stocks J, Moore M, Wright M. Open Roux-en-Y gastric bypass in 925 patients without mortality. Am J Surg 2005;189:352-356

5. Hubens G, Balliu L, Ruppert M, Gypen B, Van TT, Vaneerdeweg W. Roux-en-Y gastric bypass procedure performed with the da Vinci robot system: is it worth it? Surg Endosc 2008;22:1690-1696 
6. Gass M, Beglinger C, Peterli R. Metabolic surgery-principles and current concepts. Langenbecks Arch Surg 2011;396:949-972

7. Laferrere B. Do we really know why diabetes remits after gastric bypass surgery? Endocrine 2011;40:162-167

8. Alfonso T, Rami L, Naji A, William O. R. Is Roux-en-Y Gastric Bypass Surgery the Most Effective Treatment for Type 2 Diabetes Mellitus in Morbidly Obese Patients? J Gastrointest Surg 2005;9:1112-1118

9. Dixon JB, Zimmet P, Alberti KG, Rubino F. Bariatric surgery: an IDF statement for obese Type 2 diabetes. Diabetic Med 2011;28:628-642

10. Ramos AC, Galvao MP, de Souza YM, Galvao M, Murakami AH, Silva AC, Canseco EG, Santamaria R, Zambrano TA. Laparoscopic duodenal-jejunal exclusion in the treatment of type 2 diabetes millitus in patients with BMI $<30 \mathrm{~kg} / \mathrm{m}^{2}$ (LBMI). Obes Surg 2009;19:307-312

11. Mistry SB, Omana JJ, Kini S. Rat models for bariatric surgery and surgery for type 2 diabetes mellitus. Obes Surg 2009;19:655-660

12. Goto Y, Kakizaki M, Masaki N. Production of spontaneous diabetic rats by repetition of selective breeding. Tohoku J Exp Med 1976;119: 85-90

13. Marion LV, Serena C, Michael RR, Nayyar I. Narrative Review: Effect of Bariatric Surgery on Type 2 Diabetes Mellitus. Ann Intern Med 2009;150:94-103

14. Rubino F, Gagner M, Gentileschi P. The early effect of the Rouxen-Y gastric bypass on hormones involved in body weight regulation and glucose metabolism. Ann Surg 2004;240:236-242

15. Ashrafian H, Athanasiou T, Li JV, Bueter M, Ahmed K, Nagpal K, Holmes E, Darzi A, Bloom S R. Diabetes resolution and hyperinsulinaemia after metabolic Roux-en-Y gastric bypass. Obesity Reviews 2011;12:e257-e272

16. de Haan BJ, Faas MM, Spijker H, van Willigen JW, de Haan A, de Vos P. Factors influencing isolation of functional pancreatic rat islets. Pancreas 2004;29:e15-e22.

17. Al-Mashat HA, Kandru S, Liu RK, Behl Y, Desta T, Graves DT. Diabetes enhances mRNA levels of proapoptotic genes and caspase activity, which contribute to impaired healing. Diabetes 2006;55: 487-495

18. Teruel MN, Chen W, Persechini A, Meyer T. Differential codes for free $\mathrm{Ca}^{2+}$-calmodulin signals in nucleus and cytosol. Curr Biol 2000;10:86-94

19. Barg S, Galvanovskis J, Gopel SO, Rorsman P, Eliasson L. Tight coupling between electrical activity and exocytosis in mouse glucagon-secreting alpha-cells. Diabetes 2000;49:1500-1510

20. Zhang ZY, Pan LJ, Zhang ZM. Functional interactions among STIM1, Orail and TRPC1 on the activation of SOCs in HL-7702 cells. Amino acids 2010;39:195-204

21. Butler AE, Janson J, Soeller WC, Butler PC. Increased-cell apoptosis prevents adaptive increase in-cell mass in mouse model of Type 2 Diabetes evidence for role of islet amyloid formation rather than direct action of amyloid . Diabetes 2003;52:2304-2314

22. Wagner J, Keizer J. Effects of rapid buffers on $\mathrm{Ca}^{2+}$ diffusion and $\mathrm{Ca}^{2+}$ oscillation. Biophys J 1994;67:447-456

23. Ashburner M, Ball CA, Blake JA, Botstein D, Butler H, Cherry JM, Davis AP, Dolinski K, Dwight SS, Eppig JT, Harris MA, Hill
DP, Issel-Tarver L, Kasarskis A, Lewis S, Matese JC, Richardson JE, Ringwald M, Rubin GM, Sherlock G. Gene Ontology: tool for the unification of biology. Nat Genet 2000;25:25-29

24. Berridge MJ, Lipp P, Bootman MD. The versatility and universality of calcium signaling. Nat Rev Mol Cell Biol 2000;1:11-21.

25. Bootman MD, Collins TJ, Peppiatt CM, Prothero LS, MacKenzie L, De Smet P, Travers M, Tovey SC, Seo JT, Berridge MJ, Ciccolini F, Lipp P. Calcium signalling: an overview. Semin Cell Dev Biol 2001;12 :3-10

26. Pories WJ, Albrecht RJ. Etiology of type II diabetes mellitus: role of the foregut. World J Surg 2001;25:527-531

27. Zhang JV,Ren PG,Avsian-Kretchmer O, Luo CW, Rauch R, Klein C, Hsueh AJW. Obestatin, a peptide encoded by the ghrelin gene, opposes ghrelin's effects on food intake. Science 2005;310:996999

28. Ikeda Y, Suehiro T, Nakamura T, Kumon Y, Hashimoto K. Clinical significance of the insulin resistance index as assessed by homeostasis model assessment. Endocr J 2001;48:81-86

29. Ukkola O. Gastric bypass and glucose metabolism. Intern Med J 2009;39:502-505

30. Thaler JP, Cummings DE. Hormonal and metabolic mechanisms of diabetes remission after gastrointestinal surgery. Endocrinology 2009:150:2518-2525

31. Engstrom BE, Burman P, Holdstock C, Karlsson FA. Effects of growth hormone $(\mathrm{GH})$ on ghrelin, leptin, and adiponectin in GH-deficient patients. J Clin Endocrinol Metab 2003;88 :5193-5198

32. Kojima M, Hosoda H, Date Y, Tonon MC, Chartrel N, Cortes CL. Ghrelin is a growth-hormone-releasing acylated peptide from stomach. Nature 1999;402:656-660

33. Meijer RI, van Wagensveld BA, Siegert CE, Eringa EC, Serne EH, Smulders YM. Bariatric surgery as a novel treatment for Type 2 Diabetes Mellitus. Arch Surg 2011;146: 744-750.

34. José MR, Silvia S, Xenia C, Sonia P, Albert G, David B, Lourdes T, Manuel P, Luis G. Effect of Roux-en-Y Gastric Bypass vs Sleeve Gastrectomy on Glucose and Gut Hormones: a Prospective Randomised Trial. J Gastrointest Surg 2012;16:1116- 1122

35. De Block CE, Van Gaal LF. GLP-1 receptor agonists for type 2 diabetes. Lancet 2009;374:4-6.

36. Rutter GA, Tsuboi T, Ravier MA. $\mathrm{Ca}^{2+}$ microdomains and the control of insulin secretion. Cell Calcium 2006;40:539-551

37. Hao MM, Li X, Rizzo MA, Rocheleau JV, Dawant BM, Piston DW. Regulation of two insulin granule populations within the reserve pool by distinct calcium sources. J Cell Sci 2005; 118:5873-5884.

38. Scheenen WJ, Wollheim CB, Pozzan T, Fasolato C. $\mathrm{Ca}^{2+}$ depletion from granules inhibits exocytosis. A study with insulin-secreting cells. J Biol Chem 1998;273: 19002-19008.

39. Worley JF, McIntyre MS, Spencer B, Mertz RJ, Roe MW, Dukes ID. Endoplasmic reticulum calcium store regulates membrane potential in mouse islet beta-cells. J Biol Chem 1994;269:1435914362

40. Min Zhang, Goforth P, Bertram R, Sherman A, Satin L. The $\mathrm{Ca}^{2+}$ dynamics of isolated mouse $\beta$-cells and islets: implications for mathematical models. Biophys J 2003;84: 2852-2870 